\title{
COHOMOLOGY OPERATIONS ON $p$-FOLD SUMS
}

\author{
EMER.Y THOMAS ${ }^{1}$
}

\begin{abstract}
A formula is given for evaluating higher order cohomology operations on integral classes that are $p$-fold multiples, $p$ a prime.
\end{abstract}

Let $\Omega$ be an $n$th order ( $n \geqq 2$ ) cohomology operation defined on integral cohomology classes, given by a relation

$$
\sum_{i} \alpha_{i} \Phi_{i}=0
$$

where each $\Phi_{i}$ is an (n-1)st order (integral) operation and each $\alpha_{i}$ is an element of the mod $p$ Steenrod algebra $\mathscr{A}$. We will think of each operation $\Phi_{i}$ as being defined on integral classes of some fixed degree, $q$. We suppose that $\Phi_{i}$ is the suspension of an operation $\Psi_{i}$, defined on classes of degree $q+1$, and that relation (1) desuspends. In particular, each $\Phi_{i}$ is additive. Suppose now that $X$ is a space and $u \in H^{q}(X, Z)$ is a class such that $\Phi_{i}(u)$ is defined for each $i$. Of course $\Omega$ is not necessarily defined on $u$, but $\Omega$ is defined on $p u$, since each $\Phi_{i}$ is additive. Our problem is: how does one compute $\Omega(p u)$ ?

In [2] a morphism $\varepsilon: \mathscr{A} \rightarrow \mathscr{A}$ is defined, of degree -1 , characterized by the following properties:

(i) $\varepsilon$ is a derivation of the graded algebra $\mathscr{A}$.

(ii) If $p=2, \varepsilon\left(S q^{n}\right)=S q^{n-1}, n \geqq 1$; if $p>2, \varepsilon\left(\beta_{p}\right)=1, \varepsilon\left(P^{i}\right)=0, i \geqq 0$, where $\beta_{p}$ denotes the $\bmod p$ Bockstein. (For $p=2, \varepsilon$ is the morphism $\hat{\kappa}$ considered by Kristensen [1].)

We now can state our result.

THEOREM. Let $\Omega$ be an operation associated with relation (1) and let $u \in H^{q}(X ; Z)$ be a class in the domain of each $\Phi_{i}$. Then,

$$
(-1)^{s} \sum \varepsilon\left(\alpha_{i}\right) \Phi_{i}(u) \subset \Omega(p u),
$$

where $s=q+\operatorname{deg} \Omega$.

Received by the editors July $27,1971$.

AMS 1969 subject classifications. Primary 5534.

Key words and phrases. Cohomology operation.

${ }^{1}$ Research supported by National Science Foundation Grant GP-29073.

(C) American Mathematical Society 1972 
Proof. Let $B$ denote a universal example for the operations $\left\{\Psi_{i}\right\}$ defined on integral classes of degree $q+1$. Thus, $B$ is an $(n-1)$ st stage Postnikov system over $K(Z, q+1)$ (note [3]). By hypothesis, there are classes $\psi_{i} \in H^{*}\left(B ; Z_{p}\right)$ such that

$$
\sum(-1)^{a_{i}} \alpha_{i} \psi_{i}=0, \text { where } a_{i}=\operatorname{deg} \alpha_{i} \text {. }
$$

Suppose $d_{i}=\operatorname{deg} \psi_{i}$, and set $C=X_{i} K\left(Z_{p}, q+1+d_{i}\right)$. If we let $\psi=\left\{\psi_{i}\right\}$, we then have a map $\psi: B \rightarrow C$. Denote by

$$
\Omega C \stackrel{i}{\longrightarrow} E \stackrel{\pi}{\longrightarrow} B
$$

the principal fibration with $\psi$ as classifying map. By hypothesis, there is a class $\lambda \in H^{s+1}\left(E ; Z_{p}\right)$, thought of as a map

$$
E \stackrel{\lambda}{\rightarrow} K\left(Z_{p}, s+1\right)=K,
$$

such that $i^{*} \lambda=\alpha=\left\{\alpha_{i}\right\} \in H^{s+1}\left(\Omega C ; Z_{p}\right)$.

Now take the loops of (2); we obtain a fibration

$$
\Omega^{2} C \stackrel{i^{\prime}}{\longrightarrow} \Omega E \stackrel{\pi^{\prime}}{\longrightarrow} \Omega B,
$$

and if we denote by $\sigma$ the loop homomorphism in cohomology, then $\sigma \psi=\phi=\left(\phi_{i}\right)$, and $\sigma \lambda=\omega$, a representative for $\Omega$.

Consider the following commutative diagram:

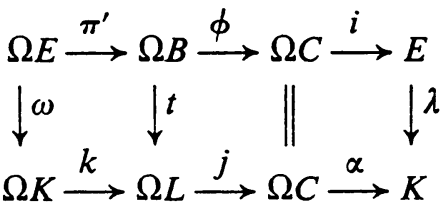

Here the lower line is the principal fibration sequence for the map $\alpha$. ( $\Omega L$ is a loop space since $\alpha$ is stable.) Since the right-hand square commutes, the map $t$ exists. Now apply the functor $[X$,$] to (3); we obtain the$ following commutative diagram with each row an exact sequence:

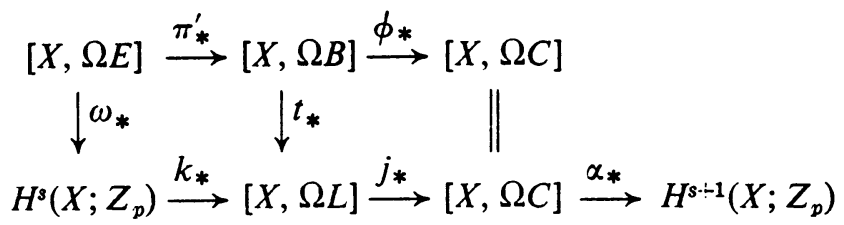

By hypothesis, there is a class $v \in[X, \Omega B]$ such that $v$ goes to $u$ in $H^{q}(X ; Z)$. Since $p[X, \Omega C]=0$, by exactness there is a class $x \in[X, \Omega E]$ such that $\pi_{*}^{\prime}(x)=p v$. And by definition $\omega_{*}(x) \in \Omega(p u)$. 
On the other hand, consider $y=t_{*}(v) \in[X, \Omega L]$. We have $j_{*}(y)=\phi_{*}(v)=$ $\left\{\phi_{i *}(v)\right\}$ where $\phi_{i *}(v) \in \Phi_{i}(u)$. Also, $k_{*} \omega_{*}(x)=p y$. Thus, by Corollary 3.7 of [2],

which completes the proof.

$$
\omega_{*}(x)=(-1)^{s} \sum_{i} \varepsilon\left(\alpha_{i}\right) \phi_{i *}(v),
$$

EXAMPLE. The simplest example of interest is the relation

$$
S q^{2} S q^{2}=0 \text {, on integral classes. }
$$

Since $\varepsilon\left(S q^{2}\right)=S q^{1}$, we then have the result:

$$
S q^{3} H^{*}(X ; Z) \subset \Omega\left(2 H^{*}(X ; Z)\right),
$$

where $\Omega$ is the secondary operation given by (4).

RemarK. As pointed out by F. Peterson, if (1) is in fact a relation that holds on $\bmod p$ classes, then $\Omega(p u) \equiv 0$. For let $\rho$ denote the cohomology homomorphism induced by the coefficient group epimorphism $Z \rightarrow Z_{p}$. Then for any integral class $v$ in the domain of $\Omega, \Omega(v) \equiv \Omega(\rho v)$, and hence

$$
\Omega(p u) \equiv \Omega(\rho(p u)) \equiv \Omega(0) \equiv 0 .
$$

\section{REFERENCES}

1. L. Kristensen, On a Cartan formula for secondary cohomology operations, Math. Scand. 16 (1965), 97-115. MR 33 \#4926.

2. L. L. Larmore and E. Thomas, Group extensions and principal fibrations, Math. Scand. (to appear).

3. C. R. F. Maunder, Cohomology operations of the Nth kind, Proc. London Math. Soc. (3) 13 (1963), 125-154. MR 35 \#2279.

Department of Mathematics, University of California, Berkeley, California 94720 